\title{
Correlation-Function Formula for the Intrinsic Viscosity of Polymer Solutions
}

\author{
Masao Dor and Koji OKano \\ Department of Applied Physics, Faculty of Engineering \\ The University of Tokyo, Bunkyo-ku, Tokyo, Japan.
}

(Received April 3, 1973)

\begin{abstract}
The correlation-function formula for the intrinsic viscosity of polymer solutions is established. It is shown that the intrinsic viscosity is made up of two parts, the first is the high-frequency-limiting viscosity $\Delta \eta_{\infty}$, independent of the frequency, and the second is the dependent part $\Delta \bar{\eta}(\omega)$. The latter part is given by the time correlation of the "reduced flux." The reduced flux is given by the averaged momentum flux with the conformation of the polymer chain fixed. The explicit expression of the reduced flux is calculated for the general model of the polymer chain, having constant bond length and constant bond angle, without using the metric tensor. It is also shown how to calculate the intrinsic viscosity based upon the stochastic local jump model.

KEY WORDS Correlation-Function Formula / Viscosity / High

Frequency / Reduced Flux / Kirkwood Model /
\end{abstract}

The object of the present paper is to establish a correlation-function formula for the intrinsic viscosity of dilute polymer solutions based upon the microscopic theory of Brownian motion. A general theory of the correlation-function formula for the viscosity of liquids has been given by a number of authors. ${ }^{1-4}$ The result is

$$
\eta(\omega)=\frac{1}{V k_{\mathrm{B}} T} \int_{0}^{\infty} \mathrm{d} t e^{-i \omega t}\langle\mathrm{~J}(t) J(0)\rangle
$$

where $\langle J(t) J(0)\rangle$ is the time correlation function of the total momentum flux $J, V$ is the volume of the liquid, $T$ is the absolute temperature and $k_{\mathrm{B}}$ is the Boltzmann constant. The microscopic expression of the total momentum flux $J$ is given by

$$
J=\sum_{j} \frac{\boldsymbol{P}_{j x} \boldsymbol{P}_{j y}}{m_{j}}+\sum_{j} \boldsymbol{r}_{j x} \boldsymbol{F}_{j y}
$$

where $m_{j}$ is the mass, $\boldsymbol{r}_{j}, \boldsymbol{p}_{j}$, are respectively the coordinate and the momentum of the molecule and $\boldsymbol{F}_{j}$ is the force acting on the molecule. Although eq 1 and 2 are quite general, it is almost impossible to calculate the correlation function without making some approximations.

Our purpose is to show how to apply the above formula to the viscosity of dilute polymer solutions. As is well known, the dynamic properties of polymer molecules are usually treated by the Brownian motion theory. The standard approach is to use a Fokker-Planck equation for the distribution function of the polymer molecules. Since the Fokker-Planck equation describes only the time evolution of the polymer configuration, we have to express the flux in terms of the coordinates of the polymer molecules. In accordance with the traditional treatment the result will be given in terms of the extra quantities, that is, the increment of the viscosity of the polymer solution to that of the solvent, will be given by the time correlation function of the excess flux such as

$$
\Delta \eta(\omega)=\frac{1}{V k_{\mathrm{B}} T} \int_{0}^{\infty} \mathrm{d} t e^{-i \omega t}\langle\Delta J(t) \Delta J(0)\rangle
$$

where $\Delta J$ is the excess flux which is expressed by the coordinate of the polymer molecule, and $\langle\Delta J(t) \Delta J(0)\rangle$ should be calculated by the FokkerPlanck equation.

Such an attempt has been performed recently by Chikahisa and Stockmayer. ${ }^{5,6}$ They presumed that $\Delta J$ is given by the following formula

$$
\Delta J=\sum_{j} R_{j x}\left(-k_{\mathrm{B}} T \frac{\partial \ln P}{\partial R_{j y}}-\frac{\partial U}{\partial R_{j y}}\right)
$$

where $R_{j x}$ is the $x$-component of the coordinate of the $j$-th unit of the polymer chain, $P$ is the 
distribution function of the polymer and $U$ is the potential energy. The expression in parentheses is the $y$-component of the thermodynamic force acting on the unit. The first term corresponds to the so-called diffusion force and the second term is the energetic force.

However two comments should be made on their expression. First, since $\Delta J$ contains the time-dependent distribution function $P\left(\boldsymbol{R}_{1}\right.$, $\left.\boldsymbol{R}_{2}, \ldots, \boldsymbol{R}_{N} ; t\right)$ in their expression, the excess momentum flux $\Delta J$ depends on time explicitly. This is quite curious because the momentum flux $J$ is a phase function and consequently $\Delta J$ should be expressed in terms of $\boldsymbol{R}_{1}, \boldsymbol{R}_{2}, \ldots$, $\boldsymbol{R}_{N}$ 's only. In this paper, we will show that $\Delta J$ does not contain the diffusion force and is expressed by only those variables describing the configuration of the polymer chain. It is shown that $\Delta J$ arises from the energetic force. At first sight it may seem that the diffusion force cannot be omitted in the case of (for example) the rigid dumbbell molecule. However this is not the case. Even in the case of the dumbbell molecule, we must consider the energetic force maintaining the bond length constant. It is shown that, by taking this force into account, the traditional results can be recovered.

The second comment is that eq 3 cannot give the finite value of the so-called limiting viscosity, which is the nonvanishing part of the intrinsic viscosity in the limit $\omega \rightarrow \infty$. Chikahisa and Stockmayer ${ }^{5}$ employed an artificial method to remove this defect in their calculation of the viscosity of the rod-like molecule. However the defect is the necessary consequence of the theory of Brownian motion. As can be seen from eq 3, the limiting viscosity, if given by eq 3 , corresponds to the time correlation function which decays in an infinitesimal time. However the Fokker-Planck equation is not applicable for such a short time scale. This point will be discussed in some detail in the following section.

In this paper we shall give the exact formal expression of $\Delta J$ based upon the microscopic Brownian motion theory. Some explicit expressions of $\Delta J$ are calculated for several models of the polymers. Especially $\Delta J$ is obtained for the general model of the polymer chain, having constant bond length and constant bond angles, without using the metric tensor. We also give a convenient expression of $\Delta J$ for the stochastic local jump model. ${ }^{16,17,18}$ This formula of $\Delta J$ seems of importance since it can be applied for the investigation of the high-frequency viscosity of the dilute polymer solutions.

\section{FORMAL EXPRESSION OF THE EXCESS FLUX}

In this section, the formal expression of $\Delta J$ is given based upon the microscopic theory of Brownian motion. ${ }^{9,10,11}$ Let us first review the theory. The present argument is based upon the projection operator method first introduced by Zwanzig. ${ }^{7,8}$ We shall first review the method in a formal way.

Consider the Hilbert space of the phase space distribution functions $|F(t)\rangle=F(x ; t)$, where $x$ denotes the point in the phase space of a complete set of the microscopic dynamical variables. Let the Hamiltonian of the system be $H(x)$. Then the equilibrium distribution function of the system is given by

$$
P_{\mathrm{eq}}(x) \propto \exp \left[-H(x) / k_{\mathrm{B}} T\right]
$$

The inner product of the two vectors in this Hilbert space is defined as

$$
\begin{aligned}
\langle F \mid G\rangle & =\int \mathrm{d} x F(x) G(x) \\
& =\langle\boldsymbol{G} \mid F\rangle
\end{aligned}
$$

The theory of the Brownian motion is based upon the assumption that there exist a set of the so-called gross variables whose time evolution is much slower than that of the other microscopic variables. Let $A_{1}(x), A_{2}(x), \ldots, A_{n}(x)$ be the gross variables. The entire Hilbert space contains a subspace consisting of the functions which depend on $x$ in such a way as $|F\rangle=$ $F\left(A_{1}(x), A_{2}(x), \ldots, A_{n}(x)\right)$. This subspace is spanned by the following functions which play the role of the unit vectors in the subspace.

$$
\left|\Delta_{a}\right\rangle=\delta\left(A_{1}(x)-a_{1}\right) \delta\left(A_{2}(x)-a_{2}\right) \cdots \delta\left(A_{n}(x)-a_{n}\right)
$$

where $a$ denotes the set of the variables $a_{1}, a_{2}, \ldots$, $a_{n}$.

Let us consider the probability distribution function of the gross variables $f(a ; t)$, which is 


\section{DoI and K. OKano}

derived from the distribution function of the total system $F(x ; t)$ as

$$
\begin{aligned}
f(a ; t) & =\int \mathrm{d} x \delta\left(A_{1}(x)-a_{1}\right) \cdots \delta\left(A_{n}(x)-a_{n}\right) F(x ; t) \\
& =\left\langle\Delta_{a} \mid F(t)\right\rangle
\end{aligned}
$$

To derive the kinetic equation of $f(a ; t)$, it is convenient to introduce the following projection operator

$$
\mathscr{P}=\int \mathrm{d} a\left|\tilde{\Delta}_{a}\right\rangle\left\langle\Delta_{a}\right|
$$

In the above equation $\left|\tilde{\Delta}_{a}\right\rangle$ is defined as

where

$$
\left|\tilde{\Delta}_{a}\right\rangle=\frac{P_{\mathrm{eq}}(x) \Delta_{a}(x)}{\bar{P}_{\mathrm{eq}}(a)}
$$

$$
\bar{P}_{\text {eq }}(a)=\int \mathrm{d} x \Delta_{a}(x) P_{\text {eq }}(x)=\left\langle\Delta_{a} \mid P_{\text {eq }}\right\rangle
$$

It is easy to verify the following relations

$$
\begin{aligned}
\left\langle\tilde{\Delta}_{a} \mid \Delta_{a^{\prime}}\right\rangle & =\delta\left(a-a^{\prime}\right) \\
\mathscr{P}^{2} & =\mathscr{P}
\end{aligned}
$$

where

$$
\delta\left(a-a^{\prime}\right)=\delta\left(a_{1}-a_{1}{ }^{\prime}\right) \delta\left(a_{2}-a_{2}{ }^{\prime}\right) \cdots \delta\left(a_{n}-a_{n}{ }^{\prime}\right)
$$

The time evolution of the distribution function of the total system is given by the Liouville operator $\mathscr{L}$.

$$
\frac{\partial}{\partial t}|F(t)\rangle=-\mathscr{L}|F(t)\rangle
$$

The following relation can be obtained by some algebraic manipulations.

$$
\begin{aligned}
\frac{\partial}{\partial t} \mathscr{P}|F(t)\rangle=-\mathscr{P} \mathscr{L} \mathscr{P}|F(t)\rangle \\
\quad-\int_{0}^{\infty} \mathrm{d} s \mathscr{P} \mathscr{L} e^{-s(1-\mathscr{P}) \mathscr{L}}(1-\mathscr{P}) \mathscr{L} \mathscr{P}|F(t-s)\rangle
\end{aligned}
$$

Multiplying $\left\langle\Delta_{a}\right|$ to this equation and using eq 8,9 , and 12 , we obtain

$$
\frac{\partial}{\partial t} f(a ; t)=-\int_{0}^{\infty} \mathrm{d} s \int \mathrm{d} a^{\prime} K_{a a^{\prime}}(s) f\left(a^{\prime}, t-s\right)
$$

where

$$
\begin{aligned}
K_{a a^{\prime}}(t)= & \delta(t)\left\langle\Delta_{a}|\mathscr{L}| \tilde{\Delta}_{a^{\prime}}\right\rangle \\
& +\left\langle\Delta_{a}\left|\mathscr{L} e^{-t(1-\mathscr{O}) \mathscr{L}}(1-\mathscr{P}) \mathscr{L}\right| \tilde{\Delta}_{a^{\prime}}\right\rangle
\end{aligned}
$$

This is the exact kinetic equation for $f(a ; t)$. Usually eq 17 is reduced to the Markoffian equation by invoking certain assumptions that the pertinent process is sufficiently slow, which will be discussed further later. The Markoffian equation can be written as

$$
\frac{\partial}{\partial t} f(a ; t)=-\mathscr{D} f(a ; t) \equiv \int \mathrm{d} a^{\prime} D_{a a^{\prime}} f\left(a^{\prime} ; t\right)
$$

The above argument is quite general and the operator $\mathscr{D}$ may be the diffusion operator (for the case of Brownian motion theory) or the master operator (for the case of chemical reaction problems). The problem of how eq $17 \mathrm{can}$ be reduced to eq 19 is one of the central problems in the microscopic theory of Brownian motion, and was discussed by a number of authors. Here we shall only give the physical idea underlying their discussion.

Since the gross variables vary much more slowly than the other microscopic variables, we can introduce a characteristic time $\tau_{\mathrm{c}}$ which distinguishes the gross variables from the other microscopic variables. The time $\tau_{\mathrm{c}}$ is defined so as to satisfy the following two assumptions, that is,

(i) the time variation of the gross variables can be neglected in time $\tau_{\mathrm{c}}$,

(ii) the time correlations of the other microscopic variables decay completely in time $\tau_{\mathrm{c}}$.

These assumptions lead to the Markoffian equation. To understand the meaning of these assumptions, let us consider a system which is in a nonequilibrium state at time $t=0$. The above assumptions lead to the following approximations for the time evolution of the distribution function of the system $F(x ; t)$. From the second assumption we can assume that, after the time $\tau_{\mathrm{c}}$, the system is in equilibrium for the microscopic variables but not for the gross variables. Therefore we may put

$$
|F(t)\rangle \cong \mathscr{P}|F(t)\rangle \quad\left(\text { for } t \geqq \tau_{\mathrm{c}}\right)
$$

since $\mathscr{P}|F(t)\rangle$ is the state where the system is in partial equilibrium with fixed gross variables. Further from the second assumption we can put

$$
\left|F\left(\tau_{\mathrm{c}}\right)\right\rangle \cong \mathscr{P}|F(0)\rangle
$$

since the gross variables do not vary in time $\tau_{\mathrm{c}}$. Combining eq 20 and 21 we have the following relation for time for $t \gg \tau_{\mathrm{c}}$ 


$$
\begin{aligned}
|F(t)\rangle & \cong \mathscr{P} e^{-\mathscr{S}\left(t-\tau_{\mathrm{c}}\right)}\left|F\left(\tau_{\mathrm{c}}\right)\right\rangle \\
& \cong \mathscr{P} e^{-\mathscr{S} t} \mathscr{P}|F(0)\rangle
\end{aligned}
$$

Let us now consider the time correlation function

$$
\begin{aligned}
\langle J(t) J(0)\rangle & =\int d x J(x) e^{-\mathscr{L} t} J(x) P_{\mathrm{eq}}(x) \\
& =\left\langle J\left|e^{-\mathscr{L} t}\right| J P_{\mathrm{eq}}\right\rangle
\end{aligned}
$$

By regarding $\exp [-\mathscr{L} t] J(x) P_{\text {eq }}(x)$ as $|F(t)\rangle$ in eq 22 we have

$$
\begin{aligned}
& \langle J(t) J(0)\rangle \\
& \quad \cong\left\langle J\left|\mathscr{P} e^{-\mathscr{L} t} \mathscr{P}\right| J P_{\text {eq }}\right\rangle \\
& =\int \mathrm{d} a \mathrm{~d} a^{\prime}\left\langle J \mid \tilde{\Delta}_{a}\right\rangle\left\langle\Delta_{a}\left|e^{-\mathscr{L} t}\right| \tilde{\Delta}_{a^{\prime}}\right\rangle\left\langle\Delta_{a^{\prime}} \mid J P_{\mathrm{eq}}\right\rangle
\end{aligned}
$$

The time correlation $\left\langle\Delta_{a}|\exp [-\mathscr{L} t]| \tilde{\Delta}_{a^{\prime}}\right\rangle$ is given by the diffusion operator

$$
\left\langle\Delta_{a}\left|e^{-\mathscr{L} t}\right| \tilde{\Delta}_{a^{\prime}}\right\rangle \cong\left[e^{-D t}\right]_{a a^{\prime}}
$$

where $[\exp (-D t)]_{a a^{\prime}}$ is the matrix element of the matrix $\exp (-D t)$. Eq 25 is the consequence of reducing eq 18 to 19 . Further we note the relation

$$
\left\langle\Delta_{a} \mid J P_{\mathrm{eq}}\right\rangle=\left\langle\tilde{\Delta}_{a} \mid J\right\rangle \bar{P}_{\mathrm{eq}}(a)
$$

From eq 24, 25, and 26, we obtain

$$
\begin{aligned}
\langle J(t) J(0)\rangle & \cong \int \mathrm{d} a \mathrm{~d} a^{\prime} \bar{J}(a)\left[e^{-D t}\right]_{a a^{\prime}} \bar{J}\left(a^{\prime}\right) \bar{P}_{\mathrm{eq}}\left(a^{\prime}\right) \\
& =\int \mathrm{d} a \bar{J}(a) e^{-\mathscr{T} t} \bar{J}(a) \bar{P}_{\mathrm{eq}}(a)
\end{aligned}
$$

where

$$
\bar{J}(a) \equiv\left\langle J \mid \tilde{\Delta}_{a}\right\rangle=\left[\bar{P}_{\mathrm{eq}}(a)\right]^{-1} \int \mathrm{d} x J(x) \Delta_{a}(x) P_{\mathrm{eq}}(x)
$$

The flux $\bar{J}(a)$ is the projected one on to the subspace spanned by $\Delta_{a}$. We shall call $\bar{J}(a)$ reduced flux. The reduced flux is given by taking the thermal average of the original flux for the microscopic variables with fixed gross variables. The expression of the reduced flux is the consequence of the reduction of the exact kinetic eq 17 to the Markoffian eq 18. At this point it should be stressed that eq 27 holds only for $t \geqq \tau_{\mathrm{c}}$. For $t \lesssim \tau_{\mathrm{c}}$ we cannot neglect the contribution of the time correlation coming from the microscopic variables. The complete time correlation is therefore given by adding a term which decays completely in time $\tau_{\mathrm{c}}$. To be consistent with the Markoffian assumption, the added term should be expressed by a delta function, therefore

$$
\langle J(t) J(0)\rangle=C_{0} \delta(t)+\langle\bar{J}(t) \bar{J}(0)\rangle
$$

where

$$
\langle\bar{J}(t) \bar{J}(0)\rangle=\int \mathrm{d} a \bar{J}(a) e^{-\mathscr{D} t} \bar{J}(a) \bar{P}_{\mathrm{eq}}(a)
$$

Note that $C_{0}$ cannot be given by the diffusion equation. The assumption that the time correlation of the microscopic variables can be expressed by a delta function is justified as far as we are concerned with the time scale $\omega \leq 1 / \tau_{\mathrm{c}}$. Let us now turn to the viscosity problems of the polymer solutions. Using eq 1 , we obtain

$$
\begin{gathered}
\eta(\omega)=\eta_{\infty}+\Delta \bar{\eta}(\omega) \\
\eta_{\infty}=\frac{1}{V k_{\mathrm{B}} T} \int_{0}^{\infty} \mathrm{d} t e^{-i \omega t} C_{0} \delta(t)=\frac{C_{0}}{V k_{\mathrm{B}} T} \\
\Delta \bar{\eta}(\omega)=\frac{1}{V k_{\mathrm{B}} T} \int_{0}^{\infty} \mathrm{d} t e^{-i \omega t}\langle\bar{J}(t) \bar{J}(0)\rangle \\
=\frac{1}{V k_{\mathrm{B}} T}\left\langle\bar{J} \frac{1}{i \omega+\mathscr{D}} \bar{J}\right\rangle
\end{gathered}
$$

By solving the diffusion equation of the polymer conformation we can calculate $\langle\bar{J}(t) \bar{J}(0)\rangle$ and corresponding excess viscosity $\Delta \bar{\eta}(\omega)$. However this is not the total excess viscosity of the polymer solutions since the term $\eta_{\infty}$ also contains the contributions coming from the polymer molecules. The term $\eta_{\infty}$ comes mainly from the solvent molecules, but it also includes the contribution of the polymer molecules. The latter part contributes to the intrinsic limiting viscosity $\Delta \eta_{\infty}$. Therefore we write

$$
\eta_{\infty}=\eta_{\mathrm{s}}+\Delta \eta_{\infty}
$$

where $\eta_{\mathrm{s}}$ is the viscosity of the solvent. The excess viscosity is then given

$$
\Delta \eta(\omega)=\Delta \eta_{\infty}+\Delta \bar{\eta}(\omega)
$$

This is the final result of our formal discussion.

The above argument is summarized as follows. In setting up the kinetic equation, which may be the Fokker-Planck equation or the master equation, we have implicitly assumed the existence of the characteristic time $\tau_{\mathrm{c}}$ which satisfies the two conditions (i) and (ii) given before eq 20. Then the intrinsic complex viscosity should 


\section{Dor and K. OKano}

be written in the form shown in eq 35 , for the frequency range $\omega \lesssim 1 / \tau_{\mathrm{c}}$. The frequency dependent part of the viscosity $\Delta \bar{\eta}(\omega)$ is given by the time correlation function of the reduced flux and can be calculated by solving the pertinent kinetic equation. On the other hand the frequency independent part $\Delta \eta_{\infty}$ cannot be calculated in the framework of the kinetic equation under consideration. To calculate $\Delta \eta_{\infty}$, a more detailed investigation of the short time scale motion $\left(t \leqslant \tau_{c}\right)$ is necessary. An example of calculation of $\Delta \eta_{\infty}$ will be reported in the future paper. In the remaining part of this paper, we will be concerned with only $\Delta \bar{\eta}(\omega)$ and calculate the reduced flux $\bar{J}$.

\section{APPLICATION TO THE ROUSE MODEL AND RIGID DUMBBELL MODEL}

In this section we shall calculate the reduced flux by using the general formula 28 for some particular models.

First we cosider the case of the Rouse theory, ${ }^{12}$ where the gross variables are chosen as the coordinate of the submolecules. Let $\boldsymbol{R}_{j}(j=1, \ldots$, $N)$ be the cordinate of the $j$-th monomer. Let us consider submolecules which include $n$ monomers and define their coordinates as those of the end monomers. Then the coordinate of the $a$-th submolecule is given as

$$
\boldsymbol{Q}_{a}=\boldsymbol{R}_{a n} \quad(a=1,2, \ldots, N / n)
$$

The reduced flux is then given by

$$
\begin{gathered}
\bar{J}(\{\boldsymbol{Q}\})=\left[\bar{P}_{\mathrm{eq}}(\{\boldsymbol{Q}\})\right]^{-1} \int \mathrm{d} \Gamma \prod_{a=1}^{N / n} \delta\left(\boldsymbol{Q}_{a}-\boldsymbol{R}_{a n}\right) J(\Gamma) \boldsymbol{P}_{\mathrm{eq}}(\Gamma) \\
\bar{P}_{\mathrm{eq}}(\{\boldsymbol{Q}\})=\int \mathrm{d} \Gamma \prod_{a=1}^{N / n} \delta\left(\boldsymbol{Q}_{a}-\boldsymbol{R}_{a n}\right) \boldsymbol{P}_{\mathrm{eq}}(\Gamma)
\end{gathered}
$$

where $\Gamma$ denotes the points of the total phase space, and $J(\Gamma)$ is given by eq 1 , or more explicitly,

$$
\begin{aligned}
J(\Gamma)= & \frac{1}{m} \sum_{j} p_{j x} p_{j y}-\sum_{j} r_{j x} \frac{\partial U}{\partial r_{j y}} \\
& +\frac{1}{M} \sum_{j} P_{j x} P_{j y}-\sum_{j} R_{j x} \frac{\partial U}{\partial R_{j y}}
\end{aligned}
$$

where $m, \boldsymbol{r}_{j}, \boldsymbol{p}_{j}$ are the mass, coordinate and the momentum of the solvent molecules respectively and $M, \boldsymbol{R}_{j}, \boldsymbol{P}_{j}$, are those of the polymer molecules, and $U$ is the potential energy of the system. Since we have used the canonical equilibrium distribution function for $P_{\mathrm{eq}}(\Gamma)$, $J(\Gamma) P_{\text {eq }}(\Gamma)$ is written

$$
\begin{gathered}
J(\Gamma) P_{\mathrm{eq}}(\Gamma)=-k_{\mathrm{B}} T\left[\sum_{j} p_{j x} \frac{\partial}{\partial p_{j y}}-\sum_{j} r_{j x} \frac{\partial}{\partial r_{j y}}\right. \\
\left.+\sum_{j} P_{j x} \frac{\partial}{\partial P_{j y}}-\sum_{j} R_{j x} \frac{\partial}{\partial R_{j y}}\right] P_{\mathrm{eq}}(\Gamma)
\end{gathered}
$$

Therefore the integral of eq 37 can be converted to the surface integral, and the nonvanishing terms are found to be as

$$
\begin{aligned}
\bar{J}(\{\boldsymbol{Q}\})= & k_{\mathrm{B}} T\left[\bar{P}_{\mathrm{eq}}(\{\boldsymbol{Q}\})\right]^{-1} \\
& \times \int \mathrm{d} \Gamma \prod_{a=1}^{N / n} \delta\left(\boldsymbol{Q}_{a}-\boldsymbol{R}_{n a}\right) \sum_{j=1}^{N} \boldsymbol{R}_{j x} \frac{\partial P_{\mathrm{eq}}(\Gamma)}{\partial R_{j y}} \\
= & k_{\mathrm{B}} T\left[\bar{P}_{\mathrm{eq}}(\{\boldsymbol{Q}\})\right]^{-1} \sum_{b=1}^{N / n} Q_{b x} \frac{\partial}{\partial Q_{b y}} \\
& \times \int \mathrm{d} \Gamma \prod_{a=1}^{N / n} \delta\left(\boldsymbol{Q}_{a}-\boldsymbol{R}_{n a}\right) P_{\mathrm{eq}}(\Gamma) \\
= & -\sum_{b=1}^{N / n} Q_{b x} \frac{\partial A(\{\boldsymbol{Q}\})}{\partial Q_{b y}} \\
& A(\{\boldsymbol{Q}\})=-k_{\mathrm{B}} T \ln \bar{P}_{\mathrm{eq}}(\{\boldsymbol{Q}\})
\end{aligned}
$$

where $A(\{Q\})$ is the free energy of the system under the restriction that the positions of the submolecules are fixed. Note that the reduced flux does not contain any diffusion force. The force that appears in the reduced flux is the mean force of interaction between the submolecules, which in general includes the excluded volume effect. If we neglect the excluded volume effect and assume that $\overline{\boldsymbol{P}}_{\text {eq }}(\{\boldsymbol{Q}\})$ is given by the Gaussian distribution function, then the wellknown result is immediately recovered. It should be stressed that the above result holds even when the hydrodynamic interaction has to be taken into account.

As a next example, let us consider the case of the dumbbell molecule. The purpose of this calculation is to illustrate that the diffusion force does not appear in the reduced flux even for the rigid macromolecules.

Let $\boldsymbol{R}_{1}, \boldsymbol{R}_{2}$ be the coordinates of the friction centers of the dumbbell molecule. Since the length of the dumbbell molecule is constant, there are only five degrees of freedom, that is, the position of the center of mass $\boldsymbol{R}$ and the 
angular part of the polar coordinate of the bond $\theta, \varphi$. We regard these five variables as relevant gross variables. Repeating the same argument given in deriving eq 41 , we obtain

$$
\begin{aligned}
& \bar{J}(\boldsymbol{R}, \theta, \varphi)= k_{\mathrm{B}} T\left[\bar{P}_{\mathrm{eq}}(\boldsymbol{R}, \theta, \varphi)\right]^{-1} \\
& \times \int \mathrm{d}^{3} \boldsymbol{R}_{1}{ }^{\prime} \mathrm{d}^{3} \boldsymbol{R}_{2}{ }^{\prime} \delta\left(\boldsymbol{R}-\boldsymbol{R}^{\prime}\right) \delta\left(\theta-\theta^{\prime}\right) \\
& \times \delta\left(\varphi-\varphi^{\prime}\right) \sum_{j=1}^{2} \boldsymbol{R}_{j x}^{\prime} \frac{\partial \boldsymbol{P}_{\mathrm{eq}}\left(\left\{\boldsymbol{R}^{\prime}\right\}\right)}{\partial \boldsymbol{R}_{j y}^{\prime}} \\
& \overline{\boldsymbol{P}}_{\mathrm{eq}}(\boldsymbol{R}, \theta, \varphi)= \int \mathrm{d}^{3} \boldsymbol{R}_{1}{ }^{\prime} \mathrm{d}^{3} \boldsymbol{R}_{2}{ }^{\prime} \delta\left(\boldsymbol{R}-\boldsymbol{R}^{\prime}\right) \delta\left(\theta-\theta^{\prime}\right) \\
& \times \delta\left(\varphi-\varphi^{\prime}\right) \boldsymbol{P}_{\mathrm{eq}}\left(\left\{\boldsymbol{R}^{\prime}\right\}\right)
\end{aligned}
$$

where $\boldsymbol{R}^{\prime}, \theta^{\prime}, \varphi^{\prime}$ is a function of $\boldsymbol{R}_{1}{ }^{\prime}$ and $\boldsymbol{R}_{2}{ }^{\prime}$, for example, $\boldsymbol{R}^{\prime}=\left(\boldsymbol{R}_{1}{ }^{\prime}+\boldsymbol{R}_{2}{ }^{\prime}\right) / 2$. The equilibrium distribution function $P_{\mathrm{eq}}(\{\boldsymbol{R}\})$ is given as

$$
P_{\text {eq }}(\{\boldsymbol{R}\})=\frac{1}{V} \cdot \frac{1}{4 \pi l^{2}} \delta\left(\left|\boldsymbol{R}_{1}-\boldsymbol{R}_{2}\right|-l\right)
$$

where $l$ is the bond length of the dumbbell molecule and $V$ is the volume of the polymer solution. From now on we put $V=1$, then we consider a polymer solution of unit concentration.

Now we calculate the integral of eq 44 and 45. For simplicity of notation we write eq 45 as

$$
\bar{P}_{\text {eq }}(\boldsymbol{R}, \theta, \varphi)=\int_{\boldsymbol{R}, \theta, \varphi \text { fixed }} \mathrm{d}^{3} R_{1} \mathrm{~d}^{3} R_{2} P_{\text {eq }}(\{\boldsymbol{R}\})
$$

where the comment under the integral symbol " $\boldsymbol{R}, \theta, \varphi$ fixed" means that the integral with respect to $\boldsymbol{R}, \theta, \varphi$ should not be made. This is the result of the property of the delta function in eq 45 . To calculate eq 47 we introduce a set of independent variables $\boldsymbol{R}, \theta, \varphi$ and $r$, where $r$ is the bond length. Then

$$
\mathrm{d}^{3} R_{1} \mathrm{~d}^{3} R_{2}=r^{2} \sin \theta \mathrm{d}^{3} R \mathrm{~d} r \mathrm{~d} \theta \mathrm{d} \varphi
$$

Since the integral with respect to $R, \theta, \varphi$ should not be made eq 47 becomes

$$
\begin{aligned}
\overline{\boldsymbol{P}}_{\mathrm{eq}}(\boldsymbol{R}, \theta, \varphi) & =\int_{0}^{\infty} \mathrm{d} r r^{2} \sin \theta P_{\mathrm{eq}}(\{\boldsymbol{R}\}) \\
& =\frac{1}{4 \pi l^{2}} \int_{0}^{\infty} \mathrm{d} r r^{2} \sin \theta \delta(r-l) \\
& =\frac{\sin \theta}{4 \pi}
\end{aligned}
$$

Similarly,

$$
\begin{aligned}
\bar{J}(\boldsymbol{R}, \theta, \varphi)= & k_{\mathrm{B}} T\left[\bar{P}_{\mathrm{eq}}(\boldsymbol{R}, \theta, \varphi)\right]^{-1} \\
& \times \int_{0}^{\infty} \mathrm{d} r r^{2} \sin \theta \sum_{j=1}^{2} R_{j x} \frac{\partial P_{\mathrm{eq}}(\{\boldsymbol{R}\})}{\partial R_{j y}}
\end{aligned}
$$

The following relation is easily verified

$$
\sum_{j=1}^{2} R_{j x} \frac{\partial}{\partial R_{j y}} P_{\mathrm{eq}}(\{\boldsymbol{R}\})=\frac{r n_{x} n_{y}}{4 \pi l^{2}} \frac{\partial}{\partial r} \delta(r-l)
$$

where $\boldsymbol{n}$ is the unit vector in the direction of the bond. Note that $\boldsymbol{n}$ is a function of $\theta$ and $\varphi$. Then substituting eq 51 and 49 into eq 50 , we obtain

$$
\begin{aligned}
\bar{J}(\boldsymbol{R}, \theta, \varphi) & =\frac{k_{\mathrm{B}} T}{l^{2}} \int_{0}^{\infty} \mathrm{d} r r^{3} n_{x} n_{y} \frac{\partial}{\partial r} \delta(r-l) \\
& =-\frac{3 k_{\mathrm{B}} T}{l^{2}} \int_{0}^{\infty} \mathrm{d} r r^{2} n_{x} n_{y} \delta(r-l) \\
& =-3 k_{\mathrm{B}} T n_{x} n_{y}
\end{aligned}
$$

Or more explicitly

$$
\bar{J}(\boldsymbol{R}, \theta, \varphi)=-3 k_{\mathrm{B}} T \sin ^{2} \theta \sin \varphi \cos \varphi
$$

However the expression of eq 52 is more simple and intuitive. Note that the reduced flux of eq 52 is given by the energetic force. Although there appears no potential $U\left(\boldsymbol{R}_{1}, \boldsymbol{R}_{2}\right)$ in the above calculation, the existence of such a potential is implicitly assumed because the calculation is based upon eq 44 . In fact if we consider a potential having deep minimum at $\left|\boldsymbol{R}_{1}-\boldsymbol{R}_{2}\right|=l$, for example, $U=\alpha\left(\left|\boldsymbol{R}_{1}-\boldsymbol{R}_{2}\right|-l\right)^{2}$, the calculated reduced flux coincides with eq 52 in the limit $\alpha \rightarrow \infty$. The important point is that the reduced flux is independent of the explicit form of the potential if the potential has a sufficiently deep minimum at $\left|\boldsymbol{R}_{1}-\boldsymbol{R}_{2}\right|=l$.

It is easily shown that the reduced flux given by eq 52 together with the correlation function formula of eq 33 lead to the same result as that obtained by the conventional method. Thus we have

$$
\begin{aligned}
\Delta \bar{\eta}(\omega)= & \frac{1}{k_{\mathrm{B}} T} \int \mathrm{d}^{3} R \mathrm{~d} \theta \mathrm{d} \varphi \frac{\sin \theta}{4 \pi} \bar{J}(\boldsymbol{R}, \theta, \varphi) \\
& \times \frac{1}{i \omega+\mathscr{D}} \overline{\boldsymbol{J}}(\boldsymbol{R}, \theta, \varphi)
\end{aligned}
$$

In the above equation $\mathscr{D}$ is the diffusion operator

$$
\mathscr{D}=D_{\mathrm{t}} \frac{\partial^{2}}{\partial \boldsymbol{R}^{2}}+D_{\mathrm{r}}\left(\frac{1}{\sin \theta} \frac{\partial}{\partial \theta} \sin \theta \frac{\partial}{\partial \theta}+\frac{1}{\sin ^{2} \theta} \frac{\partial^{2}}{\partial \varphi^{2}}\right)
$$


where $D_{\mathrm{t}}$ and $D_{\mathrm{r}}$ are the translational and rotational diffusion coefficient respectively. Substituting eq 52 and 55 into eq 54 and by using the relation

we obtain

$$
\mathscr{D} n_{x} n_{y}=6 D_{r} n_{x} n_{y}
$$

$$
\begin{aligned}
\Delta \bar{\eta}(\omega) & =9 k_{\mathrm{B}} T \int \frac{\mathrm{d} \theta \mathrm{d} \varphi}{4 \pi} \sin \theta n_{x}{ }^{2} n_{y}{ }^{2} \frac{1}{i \omega+6 D_{\mathrm{r}}} \\
& =\frac{3 k_{\mathrm{B}} T}{5} \frac{1}{i \omega+6 D_{\mathrm{r}}}
\end{aligned}
$$

This result agrees with that obtained by the traditional method ${ }^{13}$ except that the limiting viscosity is not given in this calculation.

\section{CALCULATION OF THE REDUCED FLUX FOR THE GENERAL MODEL}

In this section we shall consider the general model of the polymer chain investigated by Kirkwood $^{14,15}$ and others. This model is perhaps the most realistic one. The bond length and the bond angle are held constant and equal to $l$ and $\theta$ respectively. There exists a potential of hindered rotation. In this model the relevant gross variables are the internal rotation angles $\varphi_{1}, \varphi_{2}, \ldots$ To determine the conformation of the chain completely, other variables are necessary such as the coordinates of the initial monomer and the first and second bond vectors. However since the polymer chain is long enough, the effect of these coordinates of the end monomers will not be of importance. Therefore we may assume that these variables are fixed, and we do not take them into account explicitly. We will obtain the result in a form which does not depend on these variables explicitly.

Let $\boldsymbol{R}^{1}, \boldsymbol{R}^{2}, \ldots, \boldsymbol{R}^{N}$ be the position of the monomers. In this calculation we use the upper suffix to denote the monomers and lower suffix to denote the three components of a vector such as, for example, $R_{x}{ }^{1}, R_{y}{ }^{1}, R_{x}{ }^{2}$. The reduced flux is given by

$$
\begin{aligned}
& \bar{J}(\{\varphi\})=k_{\mathrm{B}} T\left[\bar{P}_{\mathrm{eq}}(\{\varphi\})\right]^{-1} \\
& \quad \times \int_{\varphi^{1}, \varphi^{2}, \ldots \text { fixed }} \Pi \mathrm{d}^{3} R^{a} \sum_{b=1}^{N} R_{x}{ }^{b} \frac{\partial P_{\mathrm{eq}}(\{\boldsymbol{R}\})}{\partial R_{y}{ }^{b}} \\
& \bar{P}_{\text {eq }}(\{\varphi\})=\int_{\varphi^{1}, \varphi^{2}, \ldots \text { fixed }} \prod_{a=1}^{N} \mathrm{~d}^{3} R^{a} P_{\mathrm{eq}}(\{\boldsymbol{R}\})
\end{aligned}
$$

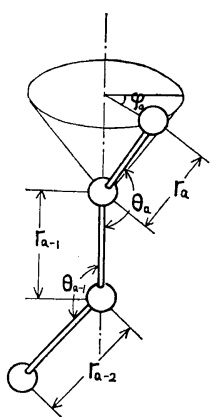

Figure 1. The definition of the independent variables. $r_{a}$; bond length, $\theta_{a}$; bond angle, and $\varphi_{a}$; internal rotation angle.

It is convenient to take the bond vectors $r^{a}=$ $\boldsymbol{R}^{a}-\boldsymbol{R}^{a-1}$ as independent variables. By using the relation

$$
\sum_{a} R_{x}^{a} \frac{\partial}{\partial R_{y}^{a}}=\sum r_{x}^{a} \frac{\partial}{\partial r_{y}^{a}}
$$

and

$$
\prod_{a} \mathrm{~d}^{3} R^{a}=\prod_{a} \mathrm{~d}^{3} r^{a}
$$

we obtain

$$
\begin{aligned}
\widetilde{J}(\{\varphi\})= & k_{\mathrm{B}} T\left[\overline{\boldsymbol{P}}_{\mathrm{eq}}(\{\varphi\})\right]^{-1} \\
& \times \int_{\varphi^{1}, \varphi^{2}, \ldots \mathrm{fixed}} \prod_{a} \mathrm{~d}^{3} r^{a} \sum_{b} r_{x}^{b} \frac{\partial P_{\mathrm{eq}}(\{\boldsymbol{R}\})}{\partial r_{y}^{b}}
\end{aligned}
$$

Let us introduce a new set of independent variables $\left(r^{a}, \theta^{a}, \varphi^{a}\right) a=1,2, \ldots$, where $r^{a}$ is the length of the bond vector and $\theta^{a}$ is the bond angle and $\varphi^{a}$ is the internal rotation angle (see Figure 1). Therefore eq 62 is transformed

$$
\begin{aligned}
\bar{J}(\{\varphi\})= & k_{\mathrm{B}} T\left[\overline{\boldsymbol{P}}_{\mathrm{eq}}(\{\varphi\})\right]^{-1} \\
& \times \int \prod_{a} \mathrm{~d} r^{a} \mathrm{~d} \theta^{a} G \sum_{b} r_{x}{ }^{b} \frac{\partial P_{\mathrm{eq}}(\{\boldsymbol{R}\})}{\partial r_{y}{ }^{b}}
\end{aligned}
$$

where $G$ is the Jacobian

$$
\boldsymbol{G}=\frac{\partial\left(\boldsymbol{r}^{1}, \boldsymbol{r}^{2}, \ldots\right)}{\partial\left(r^{1}, \theta^{1}, \varphi^{1}, r^{2}, \theta^{2}, \varphi^{2}, \ldots\right)}
$$

The equilibrium distribution function $\boldsymbol{P}_{\mathrm{eq}}(\{\boldsymbol{R}\})$ is given by using the above independent variables as

$$
P_{\mathrm{eq}}(\{\boldsymbol{R}\})=C\left[\prod_{a} \delta\left(r^{a}-l\right) \delta\left(\theta^{a}-\theta\right)\right] \mathrm{e}^{-U(\{\varphi\}) / k_{\mathrm{B}} T}
$$

where $U(\{\varphi\})$ is the potential of the hindered rotation and $C$ is the normalization constant. 
For simplicity of notation we introduce the following two dimensional vectors

$$
\boldsymbol{q}^{a}=\left(r^{a}, \theta^{a}\right)
$$

Then we have

$$
\begin{aligned}
\bar{J}(\{\varphi\})= & k_{\mathrm{B}} T\left[\bar{P}_{\mathrm{eq}}(\{\varphi\})\right]^{-1} \\
& \times \int_{a} \prod^{2} q^{a} G \sum_{a, b} r_{x}^{a}\left(\frac{\partial P_{\mathrm{eq}}}{\partial \boldsymbol{q}^{b}} \cdot \frac{\partial \boldsymbol{q}^{b}}{\partial r_{y}{ }^{a}}\right. \\
& \left.+\frac{\partial P_{\mathrm{eq}}}{\partial \varphi^{b}} \frac{\partial \varphi^{b}}{\partial r_{y}{ }^{a}}\right)
\end{aligned}
$$

By performing the partial integration for the first term, we obtain

$$
\begin{aligned}
\tilde{J}(\{\varphi\})= & k_{\mathrm{B}} T\left[\bar{P}_{\mathrm{eq}}(\{\varphi\})\right]^{-1} \\
& \times \int_{a} \prod^{2} q^{a} \sum_{a, b}\left[-\frac{\partial}{\partial \boldsymbol{q}^{b}}\left(G r_{x}{ }^{a} \frac{\partial \boldsymbol{q}^{b}}{\partial r_{y}{ }^{a}}\right)\right. \\
& \left.-\frac{G}{k_{\mathrm{B}} T} r_{x}^{a} \frac{\partial U}{\partial \varphi^{b}} \frac{\partial \varphi^{b}}{\partial r_{y}{ }^{a}}\right] P_{\mathrm{eq}} \\
= & -k_{\mathrm{B}} T\left[\bar{P}_{\mathrm{eq}}(\{\varphi\})\right]^{-1} \\
& \times \int_{a} \mathrm{~d}^{2} q^{a} \sum_{a, b}\left[\frac{\partial r_{x}{ }^{a}}{\partial \boldsymbol{q}^{b}} \cdot \frac{\partial \boldsymbol{q}^{b}}{\partial r_{y}{ }^{a}}+r_{x}^{a} \frac{\partial}{\partial \boldsymbol{q}^{b}}\left(\frac{\partial \boldsymbol{q}^{b}}{\partial r_{y}{ }^{a}}\right)\right. \\
& +r_{x}^{a} \sum_{c, \alpha} \frac{\partial \boldsymbol{q}^{b}}{\partial r_{y}{ }^{a}} \frac{\partial}{\partial r_{\alpha}{ }^{c}}\left(\frac{\partial r_{\alpha}{ }^{c}}{\partial \boldsymbol{q}^{b}}\right) \\
& \left.+\frac{r_{x}{ }^{a}}{k_{\mathrm{B}} T} \frac{\partial U}{\partial \varphi^{b}} \frac{\partial \varphi^{b}}{\partial r_{y}{ }^{a}}\right] G \boldsymbol{P}_{\mathrm{eq}}(\{\boldsymbol{R}\})
\end{aligned}
$$

where we have used the important relation

$$
\frac{\partial G}{\partial \boldsymbol{q}^{b}}=G \sum_{c, \alpha} \frac{\partial}{\partial r_{\alpha}{ }^{c}}\left(\frac{\partial r_{\alpha}^{c}}{\partial \boldsymbol{q}^{b}}\right)
$$

In the above equation the derivative $\partial / \partial \boldsymbol{q}^{b}$ should be taken by regarding $\left(r^{a}, \theta^{a}, \varphi^{a}\right)(a=1,2, \ldots)$ as independent variables and the derivative $\partial / \partial r_{\alpha}{ }^{c}$ should be taken by regarding $\boldsymbol{r}^{a}(a=1,2, \ldots)$ as independent variables, and the suffix $\alpha$ takes the values $\alpha=x, y, z$. The proof of eq 69 is given in Appendix I.

By using the property of the delta function, the integral of eq 68 can be easily carried out. The result is

$$
\begin{gathered}
\bar{J}(\{\varphi\})=\bar{J}_{r}(\{\varphi\})+\bar{J}_{\theta}(\{\varphi\})+\bar{J}_{\varphi}(\{\varphi\}) \\
\bar{J}_{r}(\{\varphi\})=-k_{\mathrm{B}} T \sum_{a, b}\left\{\frac{\partial r_{x}^{a}}{\partial r^{b}} \frac{\partial r^{b}}{\partial r_{y}{ }^{a}}+r_{x}^{a} \frac{\partial}{\partial r^{b}}\left(\frac{\partial r^{b}}{\partial r_{y}^{a}}\right)\right. \\
\left.+r_{x}^{a} \frac{\partial r^{b}}{\partial r_{y}^{a}} \sum_{c, \alpha} \frac{\partial}{\partial r_{\alpha}{ }^{a}}\left(\frac{\partial r_{\alpha}{ }^{c}}{\partial r^{b}}\right)\right\} \\
\bar{J}_{\theta}(\{\varphi\})=-k_{\mathrm{B}} T \sum_{a, b}\left\{\frac{\partial r_{x}^{a}}{\partial \theta^{b}} \frac{\partial \theta^{b}}{\partial r_{y}{ }^{a}}+r_{x}^{a} \frac{\partial}{\partial \theta^{b}}\left(\frac{\partial \theta^{b}}{\partial r_{y}{ }^{a}}\right)\right.
\end{gathered}
$$

$$
\begin{aligned}
& \left.+r_{x}^{a} \frac{\partial \theta^{b}}{\partial r_{y}{ }^{a}} \sum_{c, \alpha} \frac{\partial}{\partial r_{\alpha}{ }^{c}}\left(\frac{\partial r_{\alpha}{ }^{c}}{\partial \theta^{b}}\right)\right\} \\
& \bar{J}_{\varphi}(\{\varphi\})=-\sum_{a, b} r_{x}{ }^{a} \frac{\partial U}{\partial \varphi^{b}} \frac{\partial \varphi^{b}}{\partial r_{y}{ }^{a}}
\end{aligned}
$$

In the above equation we must put $r^{a}=l, \theta^{a}=\theta$ $(a=1,2, \ldots)$ after the derivatives are taken. The remaining task is to calculate the derivatives. The calculation is rather tedious but can be carried out exactly. As an example let us calculate $\bar{J}_{r}(\{\varphi\})$.

Since

$$
\frac{\partial r_{\alpha}{ }^{a}}{\partial r^{b}}=\frac{\partial r^{b}}{\partial r_{\alpha}{ }^{a}}=\delta_{a b} n_{\alpha}{ }^{a}
$$

( $\boldsymbol{n}^{a}$; unit vector parallel to the $a$-th bond.) we have the following expression for $\vec{J}_{\boldsymbol{r}}$

$$
\tilde{J}_{r}=-k_{\mathrm{B}} T \sum_{a}\left[n_{x}{ }^{a} n_{y}{ }^{a}+\ln _{x}{ }^{a} \frac{\partial n_{y}{ }^{a}}{\partial r^{a}}+\ln _{x}{ }^{a} \sum_{\alpha} n_{\alpha}{ }^{a} \frac{\partial n_{\alpha}{ }^{a}}{\partial r_{\alpha}{ }^{a}}\right]
$$

By using the relation

$$
\begin{gathered}
\frac{\partial n_{y}{ }^{a}}{\partial r^{a}}=0 \\
\left.\sum_{\alpha=x, y, z} \frac{\partial n_{\alpha}{ }^{a}}{\partial r_{\alpha}{ }^{a}}\right|_{r=l}=\frac{2}{l}
\end{gathered}
$$

we obtain

$$
\bar{J}_{r}(\{\varphi\})=-3 k_{\mathrm{B}} T \sum_{a} n_{x}{ }^{a} n_{y}{ }^{a}
$$

The simplicity of the result is rather surprising. The calculation of $\bar{J}_{\theta}$ and $\bar{J}_{\varphi}$ can be carried out in a similar manner. The detail of the calculation is given in Appendix II. The results are summarised as follows

$$
\begin{gathered}
\bar{J}(\{\varphi\})=\bar{J}_{r}(\{\varphi\})+\bar{J}_{\theta}(\{\varphi\})+\bar{J}_{\varphi}(\{\varphi\}) \\
\bar{J}_{r}(\{\varphi\})=-3 k_{\mathrm{B}} T \sum_{a} n_{x}{ }^{a} n_{y}{ }^{a} \\
\bar{J}_{\theta}(\{\varphi\})=-2 k_{\mathrm{B}} T \cot ^{2} \theta \sum_{a}\left[2 n_{x}{ }^{a} n_{y}{ }^{a}\right. \\
\left.+\sec \theta n_{x}{ }^{a}\left(n_{y}{ }^{a+1}+n_{y}{ }^{a-1}\right)\right] \\
\bar{J}_{\varphi}(\{\varphi\})= \\
\operatorname{cosec}^{2} \theta \sum_{a} \frac{\partial U}{\partial \varphi^{a}} \operatorname{cosec} \varphi^{a}\left[\left(n_{x}{ }^{a} n_{y}{ }^{a-2}\right.\right. \\
\left.+n_{y}{ }^{a-2} n_{x}{ }^{a-2}\right)+\cos \theta\left(1-\cos \varphi^{a}\right)\left(n_{x}{ }^{a} n_{y}{ }^{a-1}\right. \\
+n_{y}{ }^{a} n_{x}{ }^{a-1}+n_{x}{ }^{a-1} n_{y}{ }^{a-2}+n_{y}{ }^{a-1} n_{x}{ }^{a-2} \\
\left.+2 \cos \theta n_{x}{ }^{a-1} n_{y}{ }^{a-1}\right)-\cos \varphi^{a}\left(n_{x}{ }^{a} n_{y}{ }^{a}\right. \\
\left.\left.+n_{x}{ }^{a-2} n_{y}{ }^{a-2}\right)\right]
\end{gathered}
$$

This is the central result of this paper. The term $\bar{J}_{r}$ and $\bar{J}_{\theta}$ originate respectively from the 
forces which maintain the bond length and bond angle constant. The term $\bar{J}_{\varphi}$ arises from the internal rotation potential. If there is no internal rotation potential, that is, in the case of the free internal rotation, $\bar{J}$ becomes relatively simple

$$
\begin{aligned}
\bar{J}= & -k_{\mathrm{B}} T \sum_{a} n_{x}{ }^{a}\left[\left(3+4 \cot ^{2} \theta\right) n_{y}{ }^{a}\right. \\
& \left.+2 \cot \theta \operatorname{cosec} \theta\left(n_{y}{ }^{a+1} n_{y}{ }^{a-1}\right)\right]
\end{aligned}
$$

Further if the chain is freely jointed, we have

$$
\bar{J}(\{\theta, \varphi\})=-3 k_{\mathrm{B}} T \sum n_{x}{ }^{a} n_{y}{ }^{a}
$$

This formula is easily verified by examining the above calculation.

It should be stressed that the above formula can be also applied to the stochastic jump models which have been investigated recently. ${ }^{16,17,18}$ The stochastic jump model can be considered as a special case of the above general model, because if the rotation potential has several deep minima, the motion of the polymer chain will be as described by the stochastic jump model. Of course some averaging procedure is necessary for $\varphi^{1}, \varphi^{2}, \ldots$ to apply the above formula. This is done as follows.

In the stochastic jump model, the polymer chain jumps from one stable state to another stable state. Let us denote these stable states by a set of discrete parameters $\sigma^{1}, \sigma^{2}, \ldots$, where $\sigma^{a}$ indicates the range of the rotation angle $\varphi^{a}$

$$
\phi_{\sigma}<\varphi<\phi_{\sigma}^{\prime}
$$

The choice of the angles $\phi_{\sigma}$ and ${\phi_{\sigma}}^{\prime}$ is somewhat arbitrary, but does not affect the result. The reduced flux is now given as

$$
\bar{J}(\{\sigma\})=\frac{\int \prod_{a} \mathrm{~d} \varphi^{a}\left(\bar{J}_{r}(\{\varphi\})+\bar{J}_{\theta}(\{\varphi\})+\bar{J}_{\varphi}(\{\varphi\})\right) \bar{P}_{\mathrm{eq}}(\{\varphi\})}{\int \Pi \mathrm{d} \varphi^{a} \bar{P}_{\mathrm{eq}}(\{\varphi\})}
$$

where the integral with respect to $\varphi^{a}$ should be carried out in the range of eq 85 . Since the rotation potential has deep minima, $P_{\text {eq }}(\{\varphi\})$ is a rapidly varying function. On the other hand $\bar{J}_{r}(\{\varphi\})$ and $\bar{J}_{\theta}(\{\varphi\})$ are slowly varying functions. Therefore we may estimate these functions in their most stable state and take them out of the integral. Further we note the relation

$$
J_{\varphi}(\{\varphi\}) \bar{P}_{\mathrm{eq}}(\{\varphi\})=k_{\mathrm{B}} T \sum_{a . b} r_{x}{ }^{a} \frac{\partial \varphi^{b}}{\partial r_{y}{ }^{a}} \frac{\partial \bar{P}_{\mathrm{eq}}(\{\varphi\})}{\partial \varphi^{b}}
$$

We can also put the slowly varying function $r_{x}{ }^{a} \partial \varphi^{b} / \partial_{y}{ }^{a}$ out of the integral. Thus we have

$$
\begin{aligned}
\bar{J}(\{\sigma\})=\left[\bar{J}_{r}(\{\varphi\})+\bar{J}_{\theta}(\{\varphi\})\right]_{\varphi=\phi_{\sigma}}{ }^{m} \\
-k_{\mathrm{B}} T \sum_{a, b}\left[r_{x}^{a} \frac{\partial \varphi^{b}}{\partial r_{y}^{a}}\right]_{\varphi=\phi_{\sigma}{ }^{m}} \frac{\int_{c} \prod_{c} \mathrm{~d} \varphi^{c} \frac{\partial \bar{P}_{\mathrm{eq}}(\{\varphi\})}{\partial \varphi^{b}}}{\int \prod_{c} \mathrm{~d} \varphi^{c} \bar{P}_{\mathrm{eq}}(\{\varphi\})}
\end{aligned}
$$

where $\phi_{\sigma}{ }^{m}$ is the rotational angle at which the rotational potential takes its local minimum value. The last term can be neglected, because

$$
\begin{aligned}
\frac{\int_{c} \prod_{c} \mathrm{~d} \varphi^{c} \frac{\partial \bar{P}_{\mathrm{eq}}}{\partial \varphi^{b}}}{\int \prod_{c} \mathrm{~d} \varphi^{c} \bar{P}_{\mathrm{eq}}} & =\frac{\int \mathrm{d} \varphi \frac{\partial}{\partial \varphi} e^{-u(\varphi) / k_{\mathrm{B}} T}}{\int \mathrm{d} \varphi e^{-u(\varphi) / k_{\mathrm{B}} T}} \\
& =\frac{e^{-u(\phi) / k_{\mathrm{B}} T}-e^{-u\left(\phi^{\prime}\right) / k_{\mathrm{B}} T}}{\int \mathrm{d} \varphi e^{-u / k_{\mathrm{B}} T}} \\
& \simeq e^{-\Delta E / k_{\mathrm{B}} T} \ll 1
\end{aligned}
$$

where $u(\varphi)$ is the rotation potential (we have assumed the independent rotation; $\left.U=\sum_{a} u\left(\varphi^{a}\right)\right)$ and $\Delta E$ is the activation energy of the rotation. Therefore we finally obtain

$$
\bar{J}(\{\sigma\})=\left[\bar{J}_{r}(\{\varphi\})+\bar{J}_{\theta}(\{\varphi\})\right]_{\varphi=\phi_{\sigma}}{ }^{m}
$$

The reduced flux of the stochastic jump model is thus equal to that of the freely rotating model.

\section{DISCUSSION}

We have given the formal expression of the reduced flux which appears in the slowly varying part of the exact correlation function, and applied this formula to the correlation function formula of the viscosity of the polymer solutions. It is shown that the intrinsic viscosity is made up of two parts. The first part is given by the correlation of the reduced flux the time evolution of which is determined by either the diffusion equation or the master equation. The second part is the so-called limiting viscosity. The limiting viscosity is given by the time correlation of the microscopic variables which are 
discarded in the diffusion equation. The central assumption included in this argument is the existance of the characteristic time $\tau_{\mathrm{c}}$. The time $\tau_{\mathrm{c}}$ is defined so as to satisfy the following two assumptions; the relaxation times of the gross variables should be longer than $\tau_{\mathfrak{c}}$, and the longest relaxation time of the microscopic variables should be shorter than $\tau_{c}$. At present we cannot make any definite statement on the existance of $\tau_{\mathrm{c}}$ and its magnitude. It is largely a matter of the time scale concerned. We conjecture that there exists a characteristic time $\tau_{\mathbf{c}}$ which is of the order of the inverse of the jump frequency of the bond.

We have given explicit formulae for the reduced flux for the general model of the polymer chain and for the stochastic jump model. The result is relatively simple in the case of the stochastic jump model. The application of this formula for the investigation of the viscosity in the high frequency region will be attempted in another paper. Here it is mentioned that $\Delta \bar{\eta}(\omega)$ can be calculated exactly by expanding $(i \omega+\mathscr{D})^{-1}$ with respect to $1 / \omega$. From eq 33 we have

$$
\Delta \bar{\eta}(\omega)=\frac{-1}{V k_{\mathrm{B}} T} \sum_{n=0}^{\infty}\left(\frac{i}{\omega}\right)^{n+1}\left\langle\bar{J} \mathscr{D}^{n} \bar{J}\right\rangle
$$

The calculation of $\left\langle\bar{J} \mathscr{D}^{n} \bar{J}\right\rangle$ is possible in principle since $\mathscr{D}$ is a matrix of finite dimension.

\section{APPENDIX I. PROOF OF EQ 69}

We shall prove eq 69 in a general form. Let us consider the transformation of the independent variables.

$$
x_{k}=x_{k}\left(u_{1}, u_{2}, \ldots, u_{n}\right) \quad(k=1,2, \ldots, n)
$$

What we want to prove is the following formula; Let $G$ be the Jacobian

$$
G=\frac{\partial\left(x_{1}, x_{2}, \ldots, x_{n}\right)}{\partial\left(u_{1}, u_{2}, \ldots, u_{n}\right)}
$$

then

$$
\frac{\partial G}{\partial u_{l}}=G \sum_{m=1}^{n} \frac{\partial}{\partial x_{m}}\left(\frac{\partial x_{m}}{\partial u_{l}}\right)
$$

where the derivative $\partial / \partial u_{l}$ must be taken by regarding $u_{1}, u_{2}, \ldots, u_{n}$ as independent variables and the derivative $\partial / \partial x_{m}$ must be taken by regarding $x_{1}, x_{2}, \ldots, x_{n}$ as independent variables.

Let us consider the matrix $\left\|g_{i k}\right\|$ the $i-k$ com- ponent of which is $g_{i k}=\partial x_{i} / \partial u_{k}$. Let $\Delta_{i k}$ be the cofactor of $g_{i k}$. Since $G=\operatorname{det}\left\|g_{i k}\right\|$ then

$$
\begin{aligned}
\frac{\partial G}{\partial u_{l}} & =\sum_{i, k=1}^{n} \Delta_{i k} \frac{\partial g_{i k}}{\partial u_{l}} \\
& =G \sum_{i, k=1}^{n}\left(g^{-1}\right)_{k i} \frac{\partial g_{i k}}{\partial u_{l}}
\end{aligned}
$$

where $\left(g^{-1}\right)_{i k}$ is the component of the inverse matrix of $\left\|g_{i k}\right\|$. By using the identity

$$
\sum_{i=1}^{n} \frac{\partial x_{k}}{\partial u_{i}} \frac{\partial u_{i}}{\partial x_{l}}=\delta_{k l}
$$

we find

$$
\left(g^{-1}\right)_{k i}=\frac{\partial u_{k}}{\partial x_{i}}
$$

Therefore we have

$$
\begin{aligned}
\frac{\partial G}{\partial u_{l}} & =G \sum_{i, k} \frac{\partial u_{k}}{\partial x_{i}} \frac{\partial}{\partial u_{l}}\left(\frac{\partial x_{i}}{\partial u_{k}}\right) \\
& =G \sum_{i=1}^{n} \frac{\partial}{\partial x_{i}}\left(\frac{\partial x_{i}}{\partial u_{l}}\right)
\end{aligned}
$$

Then eq 94 is proved.

\section{APPENDIX II. CALCULATION OF $\overline{J_{\theta}}(\{\varphi\})$ AND $\overline{J_{\varphi}}(\{\varphi\})$}

For the calculation of $\bar{J}_{\theta}$ the derivative $\partial \theta^{b} / \partial r_{\alpha}{ }^{a}$ and $\partial r_{\alpha}{ }^{a} / \partial \theta^{b}$ are needed. The derivative $\partial \theta^{b} / \partial r_{\alpha}{ }^{a}$ is obtained as follows. Let us note the following relation

$$
\cos \theta^{b}=-\frac{\boldsymbol{r}^{b} \cdot \boldsymbol{r}^{b-1}}{\left|\boldsymbol{r}^{b}\right| \cdot\left|\boldsymbol{r}^{b-1}\right|}
$$

Differentiating this with respect to $r_{\alpha}{ }^{a}$, we obtain

$$
\begin{aligned}
\frac{\partial \theta^{b}}{\partial r_{\alpha}{ }^{a}}= & \frac{\delta_{a, b}}{r^{b} \sin \theta^{b}}\left(n_{\alpha}{ }^{b-1}+n_{\alpha}{ }^{b} \cos \theta^{b}\right) \\
& +\frac{\delta_{a, b-1}}{r^{b-1} \sin \theta^{b}}\left(n_{\alpha}{ }^{b}+n_{\alpha}{ }^{b-1} \cos \theta^{b}\right)
\end{aligned}
$$

The derivative $\partial r_{\alpha}^{a} / \partial \theta^{b}$ is most easily obtained as follows. Let us consider a small change of the bond angle $\delta \theta^{b}$ with other variables of $\left\{r^{a}, \theta^{a}, \varphi^{a}\right\}(a=1,2, \ldots)$ fixed. This change causes a uniform rotation of the portion of the chain consisting of the $b$-th and $(b+1)$-th, $\ldots$ monomers. Since the rotation vector is written as

$$
\delta \boldsymbol{\omega}=\frac{\boldsymbol{n}^{b} \times \boldsymbol{n}^{b-1}}{\left|\boldsymbol{n}^{b} \times \boldsymbol{n}^{b-1}\right|} \delta \theta^{b}
$$

the change of the bond vectors are 
M. DoI and K. OKano

$$
\delta \boldsymbol{r}^{a}= \begin{cases}0 & (a<b) \\ \delta \omega \times \boldsymbol{r}^{a} & (a \geqq b)\end{cases}
$$

Substituting eq 101 into eq 102, we obtain

$$
\frac{\partial r_{\alpha}{ }^{a}}{\partial \theta^{b}}= \begin{cases}0 & (a<b) \\ \frac{r^{a}}{\sin \theta^{b}}\left[\left(\boldsymbol{n}^{a} \cdot \boldsymbol{n}^{b}\right) n_{\alpha}{ }^{b-1}-\left(\boldsymbol{n}^{a} \cdot \boldsymbol{n}^{b-1}\right) n_{\alpha}{ }^{b}\right] & (a \geqq b)\end{cases}
$$

By using these formulas, we obtain the following results after some calculation

$$
\begin{gathered}
\sum_{a, b} \frac{\partial r_{x}{ }^{a}}{\partial \theta^{b}} \frac{\partial \theta^{b}}{\partial r_{y}{ }^{a}}=\sum_{a} \frac{1}{\sin ^{2} \theta} \\
\times\left(n_{x}{ }^{a-1}+n_{x}{ }^{a} \cos \theta\right)\left(n_{y}{ }^{a-1}+n_{y}{ }^{a} \cos \theta\right) \\
\sum_{a, b} r_{x}{ }^{a} \frac{\partial}{\partial \theta^{b}}\left(\frac{\partial \theta^{b}}{\partial r_{y}{ }^{a}}\right)=-\sum_{a} n_{x}{ }^{a} n_{y}{ }^{a} \\
\frac{\partial}{\partial \boldsymbol{r}^{c}} \cdot\left(\frac{\partial \boldsymbol{r}^{c}}{\partial \theta^{a}}\right)
\end{gathered}
$$

By summing up these terms we obtain eq 81 .

For the calculation of $\bar{J}_{\varphi}$ we need the derivative $\partial \varphi^{a} / \partial r_{y}{ }^{b}$, which is obtained as follows. Since $\varphi^{a}$ is the angle between the two vectors $\boldsymbol{r}^{a} \times \boldsymbol{r}^{a-1}$ and $\boldsymbol{r}^{a-1} \times \boldsymbol{r}^{a-2}$

or

$$
\cos \varphi^{a}=-\frac{\left(\boldsymbol{r}^{a} \times \boldsymbol{r}^{a-1}\right) \cdot\left(\boldsymbol{r}^{a-1} \times \boldsymbol{r}^{a-2}\right)}{\left|\boldsymbol{r}^{a} \times \boldsymbol{r}^{a-1}\right| \cdot\left|\boldsymbol{r}^{a-1} \times \boldsymbol{r}^{a-2}\right|}
$$

$$
\begin{aligned}
\cos \varphi^{a} & =\frac{\boldsymbol{n}^{a} \cdot \boldsymbol{n}^{a-2}-\left(\boldsymbol{n}^{a} \cdot \boldsymbol{n}^{a-1}\right)\left(\boldsymbol{n}^{a-1} \cdot \boldsymbol{n}^{a-2}\right)}{\left|\boldsymbol{n}^{a} \times \boldsymbol{n}^{a-1}\right| \cdot\left|\boldsymbol{n}^{a-1} \times \boldsymbol{n}^{a-2}\right|} \\
& =\boldsymbol{n}^{a} \cdot \boldsymbol{n}^{a-2} \operatorname{cosec} \theta^{a} \operatorname{cosec} \theta^{a-1}-\cot \theta^{a} \cot \theta^{a-1}
\end{aligned}
$$

The derivative $\partial \varphi^{a} / \partial r_{y}{ }^{b}$ is obtained by differentiating eq 109 with respect to $r_{y}{ }^{b}$ and also by using the formula 100 . The result is

$$
\begin{aligned}
\frac{\partial \varphi^{a}}{\partial r_{y}{ }^{a}}= & -\frac{1}{l \sin ^{2} \theta \sin \varphi^{a}}\left[-\cos \varphi^{a} n_{y}{ }^{a}\right. \\
& \left.+\cos \theta\left(1-\cos \varphi^{a}\right) n_{y}{ }^{a-1}+n_{y}{ }^{a-2}\right]
\end{aligned}
$$

$$
\begin{gathered}
\frac{\partial \varphi^{a}}{\partial r_{y}{ }^{a-1}}=-\frac{\cos \theta\left(1-\cos \varphi^{a}\right)}{l \sin ^{2} \theta \sin \varphi^{a}} \\
\quad \times\left[n_{y}{ }^{a}+2 \cos \theta n_{y}{ }^{a-1}+n_{y}{ }^{a-2}\right] \quad(111) \\
\frac{\partial \varphi^{a}}{\partial r_{y}{ }^{a-2}}=-\frac{1}{l \sin ^{2} \theta \sin \varphi^{a}} \\
\times\left[n_{y}{ }^{a}+\cos \theta\left(1-\cos \varphi^{a}\right) n_{y}{ }^{a-1}-\cos \varphi^{a} n_{y}{ }^{a-2}\right] \\
\frac{\partial \varphi^{a}}{\partial r_{y}{ }^{b}}=0 \quad(\text { if } b \neq a, a-1, a-2)
\end{gathered}
$$

Substituting these expressions into eq 73, we obtain eq 82 .

\section{REFERENCES}

1. M.S. Green, J. Chem. Phys., 22, 398 (1954).

2. R. Zwanzing, Ann. Rev. Phys. Chem., 16, 67 (1965).

3. J. L. Jackson and P. C. Mazur, Physica, 30, 2295 (1964).

4. B. U. Felderhof and P. C. Oppenheim, ibid., 31, 1441 (1965).

5. Y. Chikahisa and W. H. Stockmayer, R.P.P.P.J., XIV, 79 (1971).

6. W. H. Stockmayer, W. Gobush, Y. Chikahisa, and D. K. Carpenter, Discussion Faraday Soc. 49, 182 (1970).

7. R. Zwanzig, Phys. Rev., 124, 983 (1961).

8. R. Zwanzig, "Lectures in Theoretical Physics" Vol. 3, Ed. W.E. Britten and others, Interscience, New York, N.Y., 1961, p 106.

9. J.L. Lebowitz and P. Resebois, Phys. Rev., 139, A1101 (1965).

10. P. Mazur and I. Oppenheim, Physica, 50, 241 (1970).

11. R. Kubo, "Rep. Prog. Phys.," Institute of Physics and Physical Society London, 29 (1966).

12. R. E. Rouse, J. Chem. Phys., 21, 1272 (1953).

13. J. G. Kirkwood and D. L. Auer, ibid., 19, 281 (1951).

14. J. G. Kirkwood, Rec. Trav. Chim., 68, 649 (1949).

15. I. J. Erpenbeck and J. G. Kirkwood, J. Chem. Phys., 29, 909 (1958).

16. P. H. Verdier and W. H. Stockmayer, ibid., 36 227 (1962).

17. K. Iwata and M. Kurata, ibid., 50, 4008 (1969).

18. K. Iwata, ibid., 54, 12 (1971), 54, 1570 (1971). 